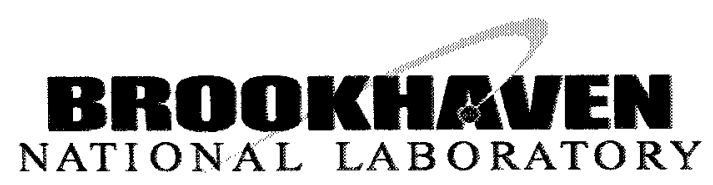

BNL-77350-2007-CP

\title{
Absolute Measurement of the Polarization of High Energy Proton Beams at RHIC
}

Y. Makdisi, A. Bravar, G. Bunce, R. Gill, H. Huang, Z. Li, A. Kponou, W. Meng, A. Nass, S. Rescia, A. Zelenski, V. Zubets, BNL, USA; M. Chapman, W. Haeberli, T. Wise, UW-Madison, USA; I.G. Alekseev, D. Svirida, ITEP, Russia; O. Jinnouchi, I. Nakagawa, RIKEN/BNL, USA; H. Okada, N. Saito, Kyoto U., Japan; S. Dhawan, Yale U., USA; E. Stephenson, IUCF, USA; $O$. Eyser, UCR, USA;

Presented at the $22^{\text {nd }}$ Particle Accelerator Conference

Albuquerque, NM

June 25-29, 2007

June 2007

\author{
Collider-Accelerator Department \\ Brookhaven National Laboratory \\ P.O. Box 5000 \\ Upton, NY 11973-5000 \\ www.bnl.gov
}

\begin{abstract}
Notice: This manuscript has been authored by employees of Brookhaven Science Associates, LLC under Contract No. DE-AC02-98CH10886 with the U.S. Department of Energy. The publisher by accepting the manuscript for publication acknowledges that the United States Government retains a non-exclusive, paid-up, irrevocable, world-wide license to publish or reproduce the published form of this manuscript, or allow others to do so, for United States Government purposes.
\end{abstract}




\section{DISCLAIMER}

This report was prepared as an account of work sponsored by an agency of the United States Government. Neither the United States Government nor any agency thereof, nor any of their employees, nor any of their contractors, subcontractors, or their employees, makes any warranty, express or implied, or assumes any legal liability or responsibility for the accuracy, completeness, or any third party's use or the results of such use of any information, apparatus, product, or process disclosed, or represents that its use would not infringe privately owned rights. Reference herein to any specific commercial product, process, or service by trade name, trademark, manufacturer, or otherwise, does not necessarily constitute or imply its endorsement, recommendation, or favoring by the United States Government or any agency thereof or its contractors or subcontractors. The views and opinions of authors expressed herein do not necessarily state or reflect those of the United States Government or any agency thereof. 


\title{
ABSOLUTE MEASUREMENT OF THE POLARIZATION OF HIGH ENERGY PROTON BEAMS AT RHIC*
}

\author{
Y. Makdisi", A. Bravar, G. Bunce, R. Gill, H. Huang, Z. Li, A. Khodinov, A Kponou, W. Meng, A \\ Nass, S. Rescia, A. Zelenski, V. Zubets, Brookhaven National Laboratory, Upton, NY 11973 \\ M.A. Chapman, W. Haeberli, T. Wise, University of Wisconsin, Madison, WI 53706 \\ 1. Alekseev, D. Svirida, Institute of Theoretical and Experimental Physics, 117259 Moscow, Russia \\ O. Jinnouchi, I. Nakagawa, RIKEN BNL Research Center, Upton, NY 11973 \\ H. Okada, N. Saito, Kyoto University, Kyoto, Japan \\ S. Dhawan, Yale University, New Haven, CT 06520 \\ E. Stephenson, Indiana University, Bloomington, IN 47408 \\ K. O. Eyser, University of California at Riverside, Riverside, CA 92521
}

\section{Abstract}

The spin physics program at the Relativistic Heavy Ion Collider (RHIC) requires knowledge of the beam polarization to better than $5 \%$. Such a goal is made the more difficult by the lack of knowledge of the analyzing power of high energy nuclear physics processes. To overcome this, a polarized hydrogen jet target was constructed and installed at one intersection region in RHIC where it intersects both beams and utilizes the precise knowledge of the jet atomic hydrogen beam polarization to measure the analyzing power in protonproton elastic scattering in the Nuclear Coulomb Interference (CNI) region at the prescribed RHIC proton beam energy. The reverse reaction is used to assess the absolute beam polarization. Simultaneous measurements taken with fast high statistics polarimeters that measure the p-Carbon elastic scattering process also in the CNI region use the jet results to calibrate the latter.

In this presentation, the status of the polarized jet target mechanics, operation, detector systems, and data analysis results are described. The statistical accuracies as well as the systematic uncertainties will be discussed.

\section{INTRODUCTION}

The polarized proton program at RHIC is designed to run at several energies. Polarimeters must be versatile enough to provide a precise measurement of the beam polarization over a wide energy range. This requires a process with a good figure of merit, a large analyzing power and a relatively high cross section. In addition, the polarimeter design should stress reduced sensitivity to inherent systematics, be non invasive as not to adversely affect the beam, and attain a high counting rate for good statistical accuracy. At RHIC we chose $p$-p and p-carbon elastic scattering at very low momentum transfer in the $\mathrm{CNI}$ region. The analyzing power, $\mathrm{A}_{\mathrm{N}}$, arises from the interference between the electromagnetic spin-flip amplitude that generates the proton anomalous magnetic moment and the hadronic spin non-flip amplitude.

In this kinematic region for pp elastic scattering, $A_{N}$ peaks at $4-5 \%$ as predicted by Schwinger in 1946 [1].
This process affords us another bonus. With identical particles, its sensitivity to whether the projectile or the target particle is polarized is the same modulo a sign change. Thus the same detectors are used to measure the scattering asymmetry from both the jet and beam.

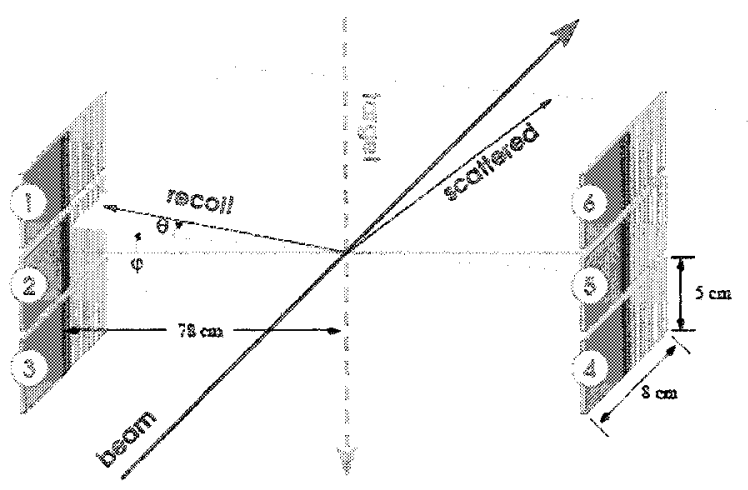

Figure1: Schematic of the scattering process in which either the Jet protons or beam protons are polarized and only the recoil protons are detected by silicon detectors.

\section{POLARIZED HYDROGEN JET TARGET}

This section describes the polarized hydrogen jet target [3-4] stressing those aspects that impact the beam polarization measurement. The jet is a structure of 9 identical differentially pumped vacuum chambers each equipped with two Varian Turbo pumps (1000 $\mathrm{l} / \mathrm{sec}$ and $10^{6}$ compression ratio for hydrogen) the exception being the first chamber with three pumps. The jet comprises several stages (figure2):

\section{The Atomic Beam Source}

Molecular hydrogen is dissociated using a variable frequency $\mathrm{RF}$ dissociator set to $21.6 \mathrm{MHz}$ and 240 watts power. With good RF matching, the reflected power was less than $2 \%$. This is followed by a $2 \mathrm{~mm}$ nozzle the neck of which is gradually cooled to about $70 \mathrm{~K}$ and a skimmer to produce a hydrogen jet that enters a series of permanent sextupole magnets with pole tip fields near $1.5 \mathrm{~T}$ arranged such that the inhomogeneous fields act to focus hydrogen atoms with electron spin $+1 / 2$ state while defocusing the

\footnotetext{
*This work was supported by Brookhaven Science Associates, LLC under Contract No. DE-AC02-98CH10886 with the U.S. Department of Energy \# makdisi@bnl.gov
} 
other and at the same time maximizing the throughput [2] and creating a waist at the point where the RHIC proton beam meets the jet.

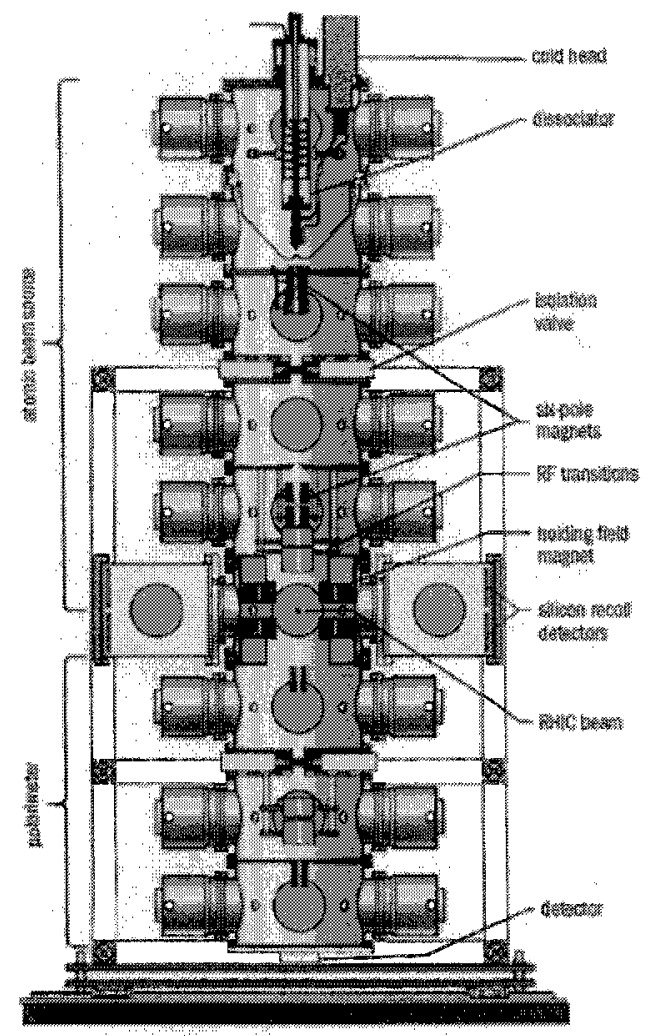

Figure2. Polarized Hydrogen jet target (detailing artwork courtesy of the CERN Courier)

\section{Radio Frequency transitions}

Two radio frequency transitions SFT and WFT at 1.43 $\mathrm{GHz}$ and $14 \mathrm{MHz}$ respectively act on the hydrogen atoms to induce a transfer from electron polarization to nuclear polarization up (SFT on, WFT off) or down (SFT off, WFT on) respectively.

\section{Holding field Magnet}

A vertical holding filed magnet using two nested and opposed Helmholtz coils provides a $1.2 \mathrm{kG}$ field over the beam-jet interacting region with $10^{-3}$ uniformity over a 4 $\mathrm{cm}$ span and assures a maximal jet polarization of $96.5 \%$. This configuration produces a zero field integral along the recoil protons path thereby preserving the energy-angle correlation seen in Fig. 4. Typically, the vacuum in this region is $\sim 10^{-8}$ Torr with the jet on and $\sim 2 \times 10^{-9}$ Torr with the valve closed. This region includes the scattering chambers on either side, the recoil proton detectors, and $\alpha$-calibration sources. A copper shield is also installed to minimize the RF impedance seen by the RHIC beam.

\section{The Jet Polarimeter}

A Breit-Rabi polarimeter measures the hydrogen beam polarization. To some extent it replicates some of the sextupole and RF transition features above, and includes special baffles and collimators. A sizable fraction of the beam, $30 \%$, is transmitted and detected by a special ion gage detector at the bottom. The RF transition efficiencies were measured to exceed $99 \%$ which greatly simplified the beam polarization measurement.

\section{The Recoil Proton spectrometer}

The pp elastic scattering process in the CNI region covers the momentum transfer $1.5 .10^{-3}<\mathfrak{t}<1.10^{-2}$ $\mathrm{GeV} / \mathrm{c}^{2}$. Forward scattered protons are not detected as their trajectory is too close to the beam direction. Recoil protons with energy range from 1-5 MeV, scatter at angles slightly less than $90^{\circ}$ in the laboratory. Two identical sets of 3 silicon strip detectors $5 \mathrm{~cm} \times 8 \mathrm{~cm}$ were placed in vacuum at a distance of $78 \mathrm{~cm}$ on either side of the beam. These were arranged in 18 channels each $4.4 \mathrm{~mm}$ wide made by connecting 40 individual strips. The two central channels near $90^{\circ}$ are not readout while the 8 channels on either side are connected to preamplifier and shaper electronics and special waveform digitizer readout that allowed to extract the time of arrival with respect to the beam crossing clock and the pulse amplitude and integral corresponding to the energy deposited in the silicon by the recoil protons (figure 1). The vertical acceptance is limited by the holding field magnet to $15^{\circ}$ and the horizontal coverage takes into account the fact that the figure of merit peaks near $t$ of $10^{-3} \mathrm{Gev} / \mathrm{c}^{2}$ and gradually decreases at higher values. Recoil protons from pp elastic scattering are identified by the scattering angle-kinetic energy correlation.

\section{JET OPERATING PARAMETERS}

The Jet operation was relatively trouble free and was continuously monitored during RHIC beam operations. A record polarized hydrogen beam intensity $12.4 \times 10^{16}$ atoms/s was measured [2-3] using the compression tube technique and also derived from the pressure rise in chamber 7 when valved off. Good intensity was attained for about 10 days before the jet nozzle started to clog up and required cleaning. The transverse jet beam size at the interaction point was measured in the lab using a $2 \mathrm{~mm}$ compression tube and later in-situ using scattered particles from the RHIC beam to be $6.6 \mathrm{~mm}$ FWHM and samples the full RHIC beam width of approximately $1-1.5 \mathrm{~mm}$. The molecular hydrogen contamination to the atomic beam was also measured off line using a quadrupole mass analyzer at $1.5 \%$ [4]. Assuming molecular hydrogen is not polarized, this represents a $3 \%$ nuclear dilution and results in an effective jet atomic beam polarization of $92.4 \mathrm{H}-$ $1.8 \%$. The jet polarization was monitored continuously during the run and averaged over each $60 \mathrm{sec}$ interval.

To reduce systematic errors, the jet polarization direction was reversed every $300 \mathrm{sec}$ using the RF transitions. Similarly, the RHIC beams alternated bunch polarization (Blue $\uparrow \downarrow$, and Yellow $\uparrow \uparrow \downarrow \downarrow$ ). The bunch number and polarization direction were tracked by the jet data acquisition system. 


\section{DATA TAKING AND ANALYSIS}

The jet was first installed in RHIC and collected its first data in 2004 which provided a precise measurement of the analyzing power of $\mathrm{pp}$ elastic scattering in the CNI region at $100 \mathrm{GeV}$ proton beam energy and confirmed the theoretical calculation [5].

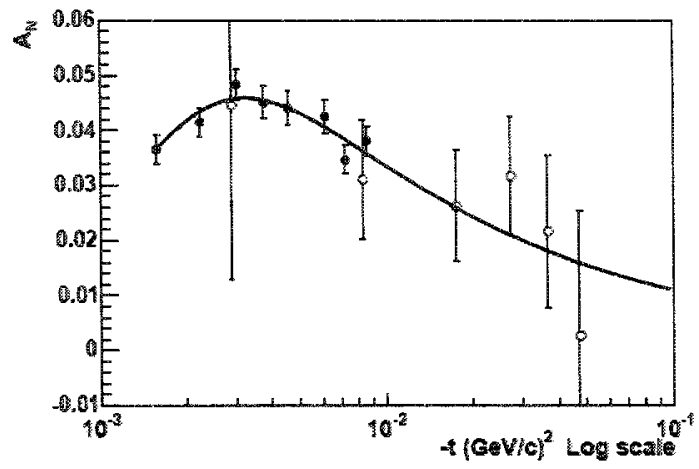

Figure3. The analyzing power measurement vs. $t$ (full circles) and data from Fermilab experiment E704 (open circles) along with the theoretical fit.

Longer runs in 2005 (this paper) and 2006 provide larger statistical samples. To date, data were collected at incident proton beam energies of $24 \mathrm{GeV}$ (RHIC injection), $31 \mathrm{GeV}$, and $100 \mathrm{GeV}$ where we measured the proton beam polarization to an accuracy of $5 \%$.

The analysis plan [6] calls for selecting the pp elastic scattering events, then measuring the resulting left-right asymmetry taking into account the jet polarization direction and averaging over the beam polarization. This and the known jet polarization provide the analyzing power of the process. The reverse process measures the asymmetry taking into account the beam polarization direction and averaging over the jet polarization. The ratio gives the average absolute beam polarization over the run.

Data analysis comprised a) calibration of the silicon strips and associated electronics ADCs for an accurate energy response. Two $\alpha$-particle sources were employed; gadolinium $3.18 \mathrm{MeV}$, and Americium 5.48 MeV. This allowed a determination of the energy loss in the silicon dead layer $(\Delta \mathrm{E} \sim 0.5 \mathrm{MeV}$ for $\alpha$-particles). For protons this loss is about a sixth of that $<0.1 \mathrm{MeV}$ which is within our resolution. b) A timing offset calibration for each channel is done from the time-of-flight vs. energy plots assuming a proton mass, Figure 4 .

Background is estimated by examining the non-signal side of the detectors or the backward scattering region. This varied with recoil energy and ranged from 4 to $10 \%$. Scattering due to the displaced beam is measured during the abort gap of the incident beam and found to be smaller by a factor of 10. Jet beam off data measures background scattering off residual gas. The background contributes to the determination of the analyzing power, but cancels out in determining the absolute beam polarization.

The square root formula where detector and luminosity asymmetries appear in second order is used to calculate the beam to jet asymmetry ratios and beam polarization.

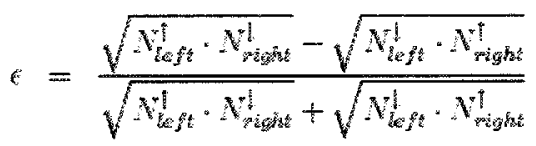

This yielded statistical uncertainties of the order of $3 \%$.
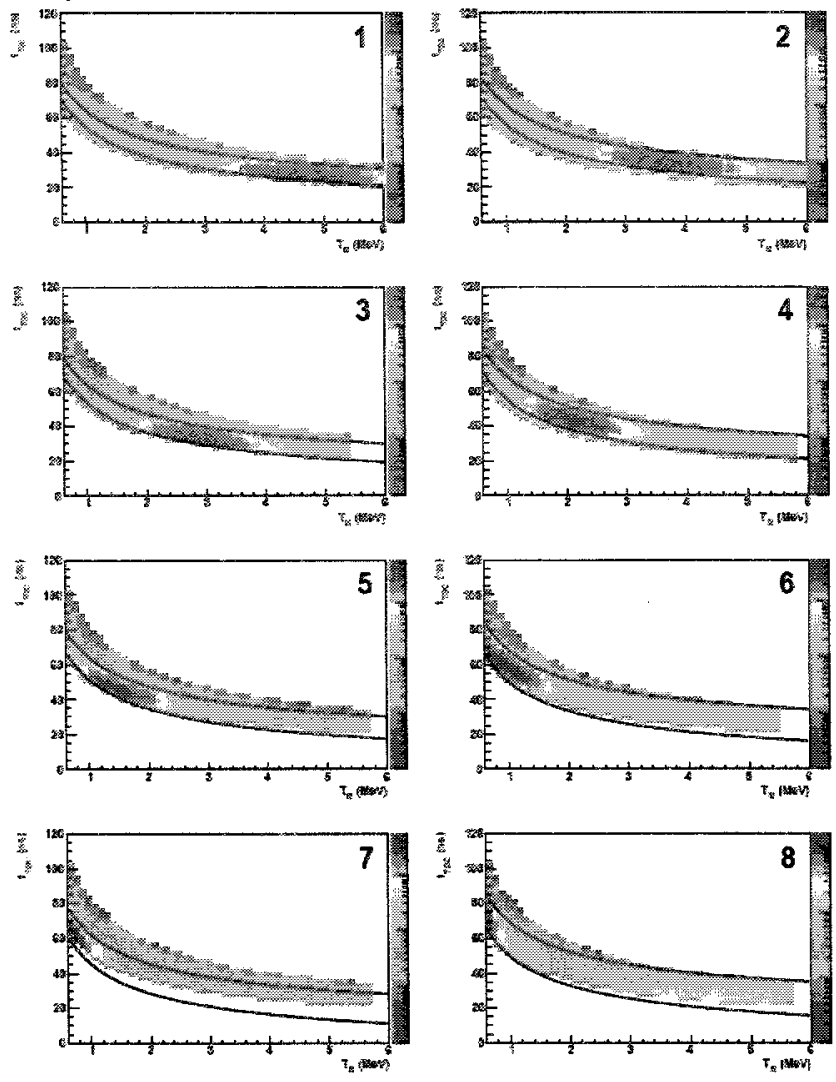

Figure4. Angle (strip \#) vs. energy correlation of the elastic scattering events.

Systematic uncertainties, amounting to $1.1 \%$ were evaluated using bunch shuffling in which each bunch is randomly assigned a spin direction. This along with the jet polarization uncertainty gave an overall systematic uncertainty $\Delta \mathrm{P}_{\text {beam }} / \mathrm{P}_{\text {beam }} \sim 2.9 \%$.

\section{SUMMARY}

The goal of measuring the beam polarization to $5 \%$ is met. $50 \%$ average beam polarization was seen in 2005 . In 2006 with increased RHIC beam intensities, the jet provided a $10 \%$ measurement of the beam polarization in an 8-hour store. Preliminary results indicate that the average beam polarizations reached $60 \%$ with few stores attaining $65 \%$.

\section{REFERENCES}

[1] J. Schwinger, Phys. Rev. 69, 681 (1946)

[2] A. Zelenski et al., NIM A536, 248 (2005)

[3] T. Wise et al., NIM A559, 1 (2006)

[4] A. Zelenski et al., SPIN2004 Conference Proceedings,

World Scientific, pp 761 (2004)

[5] H. Okada et al., Phys. Lett. B638, 450 (2006).

[6] K.O. Eyser et al., BNL C-AD Draft Analysis note

CA/AP/274, April, 2007 